This invaluable book presents an unbiased framework for modelling and using sensors to aid mobile robot navigation. It addresses the problem of accurate and reliable sensing in confined environments and makes a detailed analysis of the design and construction of a low-cost optical range finder. This is followed by a quantitative model for determining the sources and propagation of noise within the sensor. The physics behind the causes of erroneous data is also used to derive a model for detecting and labelling such data as false. In addition, the author's data processing algorithms are applied to the problem of environmental feature extraction. This forms the basis of a solution to the problem of mobile robot localisation. The book develops a relationship between the kinematics of a mobile robot during the execution of successive manoeuvres and the sensed features. Results which update a mobile vehicle's position using features from $2 D$ and $3 D$ scans are presented.

\footnotetext{
MARTIN DAVID ADAMS obtained his first degree in engineering science from Oxford University in 1988 and was conferred a DPhil in 1992 by the Robotics Research Group at the same university. After moving to Switzerland, he continued his research as a postdoctoral research assistant at the Institute of Robotics of the Swiss Federal Institute of Technology in Zurich. Between 1994 and 1995, he was a guest professor at Buchs, St Gallen (Switzerland), where he taught control theory. Since September 1996, he has been a research scientist in robotics and control at the European Semiconductor Equipment Centre (ESEC) in Cham, Switzerland. His interests include mobile robot navigation, sensor design and data interpretation, and control. He also shares a patent for a light detection and ranging sensor for use in mobile robotics.
} 
WORLD SCIENTIFIC SERIES IN ROBOTICS AND INTELLIGENT SYSTEMS

Editor-in-Charge: C J Harris (University of Southampton)

Advisor: $\quad$ T M Husband (University of Salford)

Published:

Vol. 1: Genetic Algorithms and Robotics - A Heuristic Strategy for Optimization (Y Davidor)

Vol. 2: Parallel Computation Systems for Robotics: Algorithms and Architectures (Eds. A Bejczy and A Fijany)

Vol. 3: Intelligent Robotic Planning Systems ( $P$ C- $Y$ Sheu and $Q X u e)$

Vol. 4: Computer Vision, Models and Inspection ( $A D$ Marshall and $R$ R Martin)

Vol. 5: Advanced Tactile Sensing for Robotics (Ed. H R Nicholls)

Vol. 6: Intelligent Control: Aspects of Fuzzy Logic and Neural Nets ( $C \mathrm{~J}$ Harris, $C$ G Moore and M Brown)

Vol. 7: Visual Servoing: Real-Time Control of Robot Manipulators Based on Visual Sensory Feedback (Ed. K Hashimoto)

Vol. 8: Modelling and Simulation of Robot Manipulators: A Parallel Processing Approach (A Y Zomaya)

Vol. 9: Advanced Guided Vehicles - Aspects of the Oxford AGV Project (Eds. S Cameron and P Probert)

Vol. 10: Cellular Robotics and Micro Robotic Systems ( $T$ Fukuda and T Ueyama)

Vol. 11: Recent Trends in Mobile Robots (Ed. Y F Zheng)

Vol. 12: Intelligent Assembly Systems (Eds. M Lee and J J Rowland)

Vol. 14: Intelligent Supervisory Control: A Qualitative Bond Graph Reasoning Approach ( $H$ Wang and D A Linkens)

Vol. 15: Neural Adaptive Control Technology (Eds. R Zbikowski and K J Hunt)

Vol. 17: Applications of Neural Adaptive Control Technology (Eds. J Kalkkuhl, $K J$ Hunt, $R$ Zbikowski and A Dzielinski)

Vol. 20: Obstacle Avoidance in Multi-robot Systems: Experiments in Parallel Genetic Algorithms ( $M A C$ Gill and A Y Zomaya)

Vol. 21: High-Level Feedback Control with Neural Networks (Y H Kim and F L Lewis)

Forthcoming:

Vol. 16: Advances in Robotics \& Automation for Hazardous Environment (Eds. P Lever and F Y Wang)

Vol. 18: Soft Computing in Systems and Control Technology (Ed. S Tzafestas) 
World Scientific Series in Robotics and Intelligent Systems - Vol. 13

\title{
Sensor Modelling, Design and Data Processing for Autonomous Navigation
}

\author{
Martin David Adams \\ European Semiconductor Equipment Centre \\ Cham, Switzerland
}


Published by

World Scientific Publishing Co. Pte. Ltd.

P O Box 128, Farrer Road, Singapore 912805

USA office: Suite 1B, 1060 Main Street, River Edge, NJ 07661

UK office: 57 Shelton Street, Covent Garden, London WC2H 9HE

\section{Library of Congress Cataloging-in-Publication Data \\ Adams, Martin David.}

Sensor modelling, design and data processing for autonomous navigation / Martin David Adams.

p. $\quad \mathrm{cm}$.-- (World Scientific series in robotics and

intelligent systems ; vol. 13)

Includes bibliographical references.

ISBN 9810234961 (alk. paper)

1. Mobile robots. 2. Robots -- Control systems. I. Title.

II. Series.

TJ211.415.A33 1999

$629.8^{\prime} 92--\mathrm{dc} 21$

98-36913

CIP

\section{British Library Cataloguing-in-Publication Data}

A catalogue record for this book is available from the British Library.

Copyright $(\odot) 1999$ by World Scientific Publishing Co. Pte. Ltd.

All rights reserved. This book, or parts thereof, may not be reproduced in any form or by any means, electronic or mechanical, including photocopying, recording or any information storage and retrieval system now known or to be invented, without written permission from the Publisher.

For photocopying of material in this volume, please pay a copying fee through the Copyright Clearance Center, Inc., 222 Rosewood Drive, Danvers, MA 01923, USA. In this case permission to photocopy is not required from the publisher. 


\section{Preface}

As any living being traverses its environment, information via the senses is weighted, in terms of its importance, and processed to attain some reasonable level of navigation. The ease with which animals integrate with, and travel through, the vast assortment of differing environments is still considered to be the benchmark of an automatic system to the mobile robotics researcher. Interesting experiments in which real indoor environments are scanned and the resulting data is analysed for use in navigational exercises, are used as illustrative examples throughout this book. Indeed the aim of this monograph is twofold. Firstly the problems of active sensor design, data interpretation and processing for reliable feature extraction are addressed and secondly the developed theories are applied to the problems of target tracking and navigation.

Various methods aimed at correctly interpreting real sensor data and finding algorithms for extracting useful information from this data have been documented. A survey on the vast literature assortment relating to sensor data processing and scene interpretation shows that particular subsets of the overall problem have been addressed. A coherent framework for the application oriented modelling of sensors and subsequent data processing appears to be lacking in the available literature. This book explains in detail methods for the correct interpretation of light detection and ranging sensors with the specific aim of aiding mobile robot navigation. The methods and algorithms presented are indeed applicable to a number of diverse applications, not necessarily limited to the field of autonomously guided vehicle research and the text is written to address the general problem of sensor data interpretation. Concentrating on a single application however, allows consideration to be given to actual implementations and results in some detail.

In chapter 2 , the problem of accurate and reliable sensing in confined environments is addressed and the qualities of different sensors for range estimation are compared. A key requirement in mobile robotics is high band-width, high quality sensor data as a fuel source for higher level reasoning. A detailed analysis of the electronic design of a low cost optical range finder to meet this requirement is therefore the subject of chapter 3 . The minimisation of noise sources and optimal signal processing for the production of reliable range information at the electronic level, is essential for the integration of a sensor into an autonomous navigating system. Chapter 4 presents solutions for actively scanning an environment in $3 \mathrm{D}$, from a mobile vehicle. Here the mechanical 
and optical aspects involved in the construction of a robust sensing node for autonomous navigation is discussed.

A quantitative model is examined in chapter 5 which determines the sources of noise within, and the propagation of noise through, the chosen sensing strategy. The device is theoretically modelled, to predict the type of noise distribution which can be expected under certain operating conditions. A noise model is presented and the benefits of this model are demonstrated with real $2 \mathrm{D}$ and $3 \mathrm{D}$ range scans.

Chapter 6 examines the physics behind the causes of erroneous data, which is used to derive a model to detect and label this data as false. The reliability of the model in correctly finding bad data is quantified in terms of environmental reflectance and range discontinuities. The data processing algorithms are applied to the problem of environmental feature extraction in chapter 7. A feature is defined as a pattern or event in the sensor's output data which is expected to be observable in the future, if the sensor is moved. In particular the range information and range variances, derived from the sensor noise model, are combined within an optimal filtering algorithm, capable of segmenting the range data to form edges.

Another fundamental issue from the problem of mobile robot navigation is localisation. In a further application, chapter 8 develops a relationship between the kinematics of a mobile robot, when executing successive manoeuvres, and the sensed features (edges) from the sensor. Results which update a mobile vehicle's position using features from $2 \mathrm{D}$ and 3D scans will be presented along with a discussion of the type of a priori information necessary for reliable localisation.

As a final application, the coordinates of detected edges are used in a local path planning algorithm in chapter 9. These coordinates, relative to those of the mobile robot, are tested to examine whether they can be used to attract a mobile robot towards territory which cannot be visualised or extracted from the present sensor data. Results using a real mobile vehicle and real optical range data will be presented.

Finally chapter 10 discusses the future of sensor data processing for the application of mobile robot navigation. In particular an alternative approach to mobile robot localisation is outlined. This offers a potentially, computationally economic solution which allows a moving sensor to track a single feature. The notion of a moving scanning mechanism locking onto a fixed target presents an interesting and an extant research issue and the possibility of a fully instantaneous tracking algorithm will be discussed.

Researchers in academic institutions and industry will find the sensor modelling and data processing framework useful for developing sensing systems in general. Readers at the undergraduate and graduate level interested in sensing and navigation in mobile robotics will also find the text particularly valuable. 


\section{Acknowledgments}

\section{"Seeing is believing, Sensing can be deceiving"}

During the course of the studies and experiments carried out to make this book possible, the author was greatly indebted to many colleagues for their time and cooperation.

The manuscript is based upon work carried out at the Robotics Research Group, Department of Engineering Science, University of Oxford, U.K. and at the Institute of Robotics, Swiss Federal Institute of Technology, Zurich, Switzerland.

In particular, during the doctoral dissertation work at Oxford, the author would like to thank Penny Probert, Hugh Durrant-Whyte and John Leonard for their academic and practical help and support in mobile robotics. Mike Brownlow provided invaluable inspiration from his initial electronic design work on lidar sensing.

In Zurich, this work was further supported by Prof. G. Schweitzer and major contributions to the mechanical lidar construction were provided by Matteo Camani, Stefan Mathis and Lorenz Ruf. The author further gratefully acknowledges the assistance of Daniel von Flüe with the experimental work on sonar and André Kerstens for the presented localisation and tracking algorithms with lidar. The assistance and cooperation of Philipp Bühler, Marco Fumagalli and René Schöni during many of the electronic hardware implementations and data retrieval experiments is also gratefully acknowledged.

The late night work on this book would not have been possible without McVities Hob-Nobs. 


\section{This page is intentionally left blank}




\section{Contents}

Preface $\quad$ v

Acknowledgments vii

List of Figures $\quad$ xiii

1 Introduction 1

Part I: Sensor Design and Modelling $\quad 11$

2 Range Sensing in Confined Environments 13

2.1 Introduction . . . . . . . . . . . . . . . . . . . . . 13

2.2 Stereo Vision for Range Determination . . . . . . . . . . . . . . 14

2.2 .1 Disparity between Two Images . . . . . . . . . . . . . 15

2.2.2 General Case - Camera Orientation . . . . . . . . 16

2.2.3 Calculation of Depth . . . . . . . . . . . . . 17

2.2.4 Finding Conjugate Points . . . . . . . . . . 18

2.3 Optical Sensing - Controlling the Illumination _ . . . . . . 20

2.3.1 Active Triangulation . . . . . . . . . . . . . . 20

2.3.2 Synchronised Scanning . . . . . . . . . . . . . . 22

2.3.3 Structured Light, Line Projection Systems . . . . . . . . 25

2.3.4 Light Detection and Ranging (LIDAR) . . . . . . . . 27

2.4 Ultrasonic Range Sensing . . . . . . . . . . . . . . . . . . . 28

2.4.1 Sonar - The Physics of Reflection . . . . . . . . . 30

2.5 Summary . . . . . . . . . . . . . . . . . 35

3 Lidar Sensor Design - Electronic Requirements 40

3.1 Introduction . . . . . . . . . . . . . . . . . . . 40

3.2 Lidar Range Measurement Methods . . . . . . . . . . . . . . . . 41

3.2.1 Time-of-flight Pulse Lidars . . . . . . . . . . . . . . . 41

3.2 .2 F.M.C.W. Lidars . . . . . . . . . . . . . . . . 42

3.2 .3 A.M.C.W. Lidars . . . . . . . . . . . . . . . . 43

3.3 A.M.C.W Lidar Modules . . . . . . . . . . . . . . . . . 44

3.4 Current to Light Conversion - The Transmitter Module . . . . 47 
3.5 The Receiver Module - Opto-electronic Amplification . . . . . 50

3.5.1 Light to Current Conversion . . . . . . . . . . . . 50

3.5.2 Lambertian Reflection and Signal Reception . . . . . . 56

3.5.3 Low Receiver Band-width Signal Selection . . . . . . . . 58

3.6 Dynamic Range Compression . . . . . . . . . . . . . . . 60

3.7 Frequency Reduction . . . . . . . . . . . . . . . . . . . 62

3.8 Relative Phase Discrimination . . . . . . . . . . . . . . . . . 64

3.8.1 Phase Detection . . . . . . . . . . . . . . 71

3.9 Output Filter Stage . . . . . . . . . . . . . . . . . . . 73

3.10 Received Signal Amplitude Detection . . . . . . . . . . . . 75

3.11 Summary . . . . . . . . . . . . . . . . . . 77

4 Lidar Sensor Design - Mechanical and Optical $\begin{array}{ll}\text { Requirements } & \mathbf{8 0}\end{array}$

4.1 Introduction . . . . . . . . . . . . . . . . . 80

4.2 The Scanning Mechanism - Sensor Reachability . . . . . . . 81

4.2.1 Solution 1: Twin Motor Direct Drive Mechanism . . . . 84

4.2.2 Solution 2: Single Motor with Synchronising Mechanics . . . . . . . . . . . . . . 86

4.2.3 Solution 3: Two Fixed Motors with Differential Mechanics . . . . . . . . . . . . . 87

4.3 Practical Implementation of the Scanning Mechanism . . . . . . 89

4.3.1 Practical Realisation of Solution 3 . . . . . . . . . . 89

4.3.2 Practical Realisation of Solution 1 . . . . . . . . . . . 91

4.4 Coaxial Optical Alignment . . . . . . . . . . . . . . . . . . . 92

4.5 Focusing the Received Light Signal . . . . . . . . . . . . . . . . 94

4.6 Physical Compactness and Overall Dimensions . . . . . . . . . . 97

4.7 Optical Transmitter Construction . . . . . . . . . . . . . . . 101

4.8 Summary . . . . . . . . . . . . . . . . . . . . . . 103

5 Quantitative Sensor Modelling - Noise Analysis 105

5.1 Introduction . . . . . . . . . . . . . . . . . . 105

5.2 Sensor Noise Revisited . . . . . . . . . . . . . . . . . . 107

5.2.1 Noise Propagation Within the Sensor . . . . . . . . 108

5.2.2 The Nature of the Range Distribution . . . . . . . . . . 111

5.3 Averaging Several Range Estimates . . . . . . . . . . . . . 112

5.4 Calibrating the Sensor - Results . . . . . . . . . . . . . 113

5.4.1 Amplitude Induced Phase Shifts . . . . . . . . . . . 116

5.4 .2 Range Variance . . . . . . . . . . . . . . . . 117

5.5 2D Range/Amplitude Scanning - Results . . . . . . . . . . 118

5.5.1 Temporal Range Data Averaging . . . . . . . . 120 
5.6 3D Range/Amplitude Scanning - Results . . . . . . . . . . . 121

5.7 Summary . . . . . . . . . . . . . . . . . . . . . 123

6 Qualitative Sensor Modelling - False Data 126

6.1 Introduction . . . . . . . . . . . . . . . . . 126

6.2 Discontinuous Points - Mixed Pixels . . . . . . . . . . . . . . . 128

6.3 Simultaneous Reflection of Signals from Two Targets . . . . . . 132

6.4 Discontinuity Detection . . . . . . . . . . . . . . . . 136

6.4.1 False Detection and Range Sensitivity . . . . . . . . 139

6.4.2 Surface Reflectance Sensitivity . . . . . . . . . . . . . . . 141

6.5 Practical Implementation and Results . . . . . . . . . . . . . 143

6.6 Summary . . . . . . . . . . . . . . . . . . . 147

Part II: Mobile Robot Navigation Oriented Signal Processing 151

7 Environmental Feature Extraction 153

7.1 Introduction . . . . . . . . . . . . . . . . . . . 153

7.2 Feature Extraction - Edge Detection . . . . . . . . . . . 155

7.2.1 Edge Detection - The Extended Kalman Filter . . . . . 155

7.2 .2 Validation Gate Formation . . . . . . . . . . . . . 158

7.2 .3 Filter Initialisation . . . . . . . . . . . . . . . . . . . 159

7.3 Edge Detection: Results . . . . . . . . . . . . . . . . . . 160

7.4 Summary . . . . . . . . . . . . . . . . . 166

8 Sensor Driven Mobile Robot Localisation 168

8.1 Introduction . . . . . . . . . . . . . . . . . . . 168

8.2 Mobile Robot Localisation . . . . . . . . . . . . . . . . . . . . . 171

8.2.1 Mobile Robot Kinematics - The System Model . . . . . 171

8.2.2 2D Scanning: The Observation Model . . . . . . . . . . 172

8.2.3 3D Scanning: The Observation Model . . . . . . . . . . . 173

8.3 The Localisation Cycle . . . . . . . . . . . . . . . . . . . . 173

8.4 Filter Initialisation . . . . . . . . . . . . . . . . . . . . 176

8.5 Localisation Results . . . . . . . . . . . . . . . . . . . . 177

8.5.1 Localisation with an a priori Map . . . . . . . . . . 177

8.5.2 Localisation without an a priori Map . . . . . . . . 178

8.6 3D Mobile Robot Localisation . . . . . . . . . . . . . . . . 182

8.7 Summary . . . . . . . . . . . . . . . . . . . 187

9 Application: Mobile Robot Path Planning 190

9.1 Introduction . . . . . . . . . . . . . . . . . . . . . 190

9.2 Edge — Target Relationship . . . . . . . . . . . . . . . . . 193 
9.3 Target Validity . . . . . . . . . . . . . . . . . . . . . . . . . . . 194

9.4 Target Reachability . . . . . . . . . . . . . . . . . . . . 195

9.5 Dynamic Target Production . . . . . . . . . . . . . . . . 198

9.6 Summary . . . . . . . . . . . . . . . . . . . . . . . . . . . . . . 199

10 Conclusions and Future Research Directives 200 10.1 Conclusions . . . . . . . . . . . . . . . . . . . 200

10.2 Future Research Directives . . . . . . . . . . . . . . . . . . 201

A Discontinuity Detection - Second Differential $S$ as a

$\begin{array}{ll}\text { Function of Time } & 206\end{array}$

$\begin{array}{ll}\text { Bibliography } & 209\end{array}$

$\begin{array}{ll}\text { Index } & 222\end{array}$ 


\section{List of Figures}

1.1 The block diagram structure of the book.

1.2 A photograph of "Eric" the mobile robot platform, transporting the ORS-1 Lidar.

2.1 Idealised camera geometry for stereo vision. . . . . . . . . . . 15

2.2 Transformation vectors between camera positions. . . . . . . . . 17

2.3 A one dimensional active triangulation geometry. . . . . . . . . 21

2.4 A synchronised, one dimensional active triangulation geometry. . 22

2.5 Range estimation using a lateral effect photo-diode as the receiver in a triangulation system. . . . . . . . . . . . 24

2.6 Projected light stripe geometry for synchronised stripe scanning. 25

2.7 Infra-red light sensing by modulated signal transmission. . . . . 28

$2.82 \mathrm{D}$ range and received signal amplitude scans recorded within a laboratory environment. . . . . . . . . . . . . . 29

2.9 Sonar range data recorded in a simple laboratory environment. . 30

2.10 The geometry of ultrasonic reflection in the vicinity of a wall. . 32

2.11 Sonar range data points recorded as the transducer is scanned passed two walls.

2.12 The geometry of ultrasonic reflection within the vicinity of a corner. . . . . . . . . . . . . . . . 34

2.13 Real sonar data in the vicinity of a corner. . . . . . . . . . 35

2.14 RCD's extracted from a single sonar scan. . . . . . . . . . . 36

2.15 RCD's extracted from several sonar scans. . . . . . . . . . . 37

2.16 All RCD's extracted from 15 different sensor positions superimposed upon each other. . . . . . . . . . . . . . . 38

3.1 F.M.C.W. range estimation. . . . . . . . . . . . . . 42

3.2 Range estimation by measuring the phase shift between transmitted and received signals.

3.3 The interconnection of each functional module for A.M.C.W. range estimation. . . . . . . . . . . . . . 48 48

3.4 Block diagram of the optical transmitter. . . . . . . . . . . 50

3.5 The gain and noise characteristics for the receiver's A.P.D. . . . 52

3.6 The variables which affect diffuse reflection according to Lambert's cosine law. . . . . . . . . . . . . . . . . . 
$3.7 L C$ resonant circuit models, for $10 \mathrm{MHz}$ signal selection. . . . . 58

3.8 Weak HF received signal. . . . . . . . . . . . . . . . . 61

3.9 Strong HF received signal. . . . . . . . . . . . . . . . . . . 62

3.10 Analogue multiplication of the received and reference signals . . 64

3.11 Intermediate frequency received and reference signals derived from a weak high frequency received signal. . . . . . . . . . .

3.12 Intermediate frequency received and reference signals derived from a strong high frequency received signal . . . . . . . . 66

3.13 Block diagram representation of the phase locked loop.

3.14 I.F. and P.L.L. intermediate reference and received signals and their corresponding V.C.O. outputs for a target at $2.9 \mathrm{~m}$. . . . .

3.15 I.F. and P.L.L. intermediate reference and received signals and their corresponding V.C.O. outputs for a weakly reflecting target. . . . . . . . . . . . . . . 70

3.16 I.F. and P.L.L. intermediate reference and received signals and their corresponding V.C.O. outputs for a reflective target.

3.17 Waveforms produced by the three different phase comparators available on the CMOS 4046 P.L.L, for various input waveforms.

3.18 Range and amplitude outputs for differing target reflectivities at a fixed range.

3.19 The magnified range outputs of figure 3.18 showing the a.c. noise variations in the signal. . . . . . . . . . . 75

3.20 The signals used in the homodyne, amplitude detection module.

4.1 Scanning with restricted fields of view in bearing and elevation, on a moving vehicle. . . . . . . . . . . 83

4.2 A direct drive 2 motor, 3D scanning mechanism. . . . . . . . . . 85

4.3 A single motor 3D scanning mechanism. . . . . . . . . . . . 86

4.4 A twin motor differential 3D scanning mechanism. . . . . . . . 88

4.5 A photograph showing a mechanism based on design solution $3 . \quad 89$

4.6 Two full $360^{\circ}$ scans of a laboratory. . . . . . . . . . . . . 90

4.7 A photograph showing a mechanism based on design solution 1. 92

4.8 Optical transmitter-receiver relationships for coaxial lidar design. 93

4.9 Optical misalignment between the transmission and reception axes. 95

4.10 The drop in signal strength as a function of off-axis angle for sensor to target distances of $4 \mathrm{~m}$ and $10 \mathrm{~m} . \ldots . . . . .$.

4.11 Configurations for correctly focusing the received light signal, collected by the sensor's aperture, to the receiver. . . . . . . 97

4.12 A photograph of the simple receiver focusing system. . . . . . . 98

4.13 A photograph of a compact lidar sensor. . . . . . . . . 100 
4.14 Light transmission from a fibre-optic L.E.D. . . . . . . . . . . . 101

4.15 Transmitted light columnation using two standard lenses. . . . . 102

4.16 A photograph showing the resulting transmitter construction. . 104

5.1 Phase estimation in the presence of noise. . . . . . . . . . . 109

5.2 Calibration curves for lidar range estimation and correction. . . 114

5.3 Histograms showing the effect of different coloured targets at a given range. . . . . . . . . . . . . . . 115

5.4 Least squares function fits for range variance versus signal strength. . . . . . . . . . . . . . . . . . 118

5.5 A single $360^{\circ}$ scan from a laboratory environment. . . . . . . . . 119

5.6 Corrected scan using the experimental calibration curves. . . . . 120

5.7 Temporal data averaging and the resulting range variance. . . . 121

5.8 A 3D range map showing the immediate surroundings of the sensor. . . . . . . . . . . . . . . . . . . 122

5.9 Pixel maps showing 3D albedo, range and standard deviation plots in 'Wrap around geometry' form.

5.10 Three further albedo, range and standard deviation 3D pixel maps in a more complex environment.

6.1 Graphs showing range and signal strength versus beam angle in the vicinity of an edge.

6.2 Graphs of range and signal strength versus time as the fan blades pass by the stationary sensor aperture.

6.3 Magnified views of range and signal strength estimates versus time, as the edge of a fan blade traverses the infra-red beam. . . 132

6.4 Histograms showing the mean range and range variance as the fan traverses the infra-red beam. . . . . . . . . . . . . . 133

6.5 The splitting of the transmitted signal into two returned signals of differing phase by an edge. . . . . . . . . . . . . . 134

6.6 The relationship between chosen end conditions. . . . . . . . . 137

6.7 Graphs of signal amplitude versus time as the infra-red beam traverses different end conditions. . . . . . . . . . . . 138

6.8 Second derivative $S$ versus range $R_{2}$ for various values of $R_{1}$. . 140

6.9 Surface reflectance ratio sensitivity when the range estimates are equal. . . . . . . . . . . . . . . . . . 141

6.10 Surface reflectance ratio when the range estimates are unequal. . 142

6.11 Discontinuity detection from a single infra-red scan. . . . . . . . 144

6.12 Graph of S as computed experimentally from the previous scan. 145

6.13 Discontinuity detection from a single infra-red scan. . . . . . . . 146

6.14 Uncorrected and corrected range scans using 5600 data samples. 147 
6.15 Discontinuity detection parameters versus sensor azimuth. . . . 148

6.16 Accepted and rejected data, after filtering with the detector. . . 149

7.1 The relationship between successive range readings when scanning a planar surface. . . . . . . . . . . . . . . 156

7.2 Kalman Filter edge detection. . . . . . . . . . . . . . . . 161

7.3 Graphs of range prediction and observation versus sensor azimuth. . . . . . . . . . . . . . . . 163

7.4 Kalman Filter edge detection using prefiltered data. . . . . . . . 164

7.5 Graphs of range prediction and observation versus sensor azimuth. . . . . . . . . . . . . . . . 165

7.6 Edge detection with the EKF in a more cluttered environment. . 166

8.1 Localisation by tracking a feature in 3D space. . . . . . . . . . . 172

8.2 Determining angular uncertainty from the range variance. . . . . 175

8.3 A single scan and position update during a mobile robot run. . . 178

8.4 A 2D scan and position update after the mobile robot has moved in the negative $\mathrm{x}$ direction. . . . . . . . . . . . . . . 179

8.5 2D scans, matched edges (circles (o)) and position updates as the robot traverses its environment. . . . . . . . . . . . . . 180

8.6 2D scans, matched edges and position updates as the robot continues to traverses its environment. . . . . . . . . . . . . 181

8.7 All 23 positional estimates using the map-based localisation algorithm. . . . . . . . . . . . . . . . . . . . 182

8.8 All 23 positional estimates using the localisation algorithm without any prior known map. . . . . . . . . . . . . . . . . 183

8.9 A 3D line model of a section of the mobile robot's surroundings. 184

8.10 A section of the 3D scan data after the robot has moved $80 \mathrm{~cm}$ in the negative y direction from the 'basis' scan point. . . . . . . 185

8.11 The 3D scan data from figure 8.10 and matched edges viewed along the $\mathrm{x}$ axis. . . . . . . . . . . . . . . 186

8.12 A section of the 3D scan data recorded after the mobile robot moved to the other side of the 'basis' scan point. . . . . . . . . . 187

8.13 The 3D scan data from figure 8.12 and matched edges viewed along the $\mathrm{x}$ axis. . . . . . . . . . . . . . . . . 188

8.14 A plan view of the model environment from figure 8.9, showing the robot basis position and the hand measured positions, EKF estimated positions and, centred at these points, the uncertainty ellipsi for the other two positions. . . . . . . . . . . . . 189

9.1 Choosing a target from a single edge. . . . . . . . . . . . . . . 194 
9.2 Testing the chosen target for reachability. . . . . . . . . . . . 195

9.3 Target production from edges . . . . . . . . . . . . . . 196

9.4 Target production in a cluttered environment. . . . . . . . . . 197

9.5 Mobile robot pursuing a moving target . . . . . . . . . . . . . 198

A.1 Area, time relationship for beam traversal across end conditions 206 